\title{
Performance de Geomembranas: Agentes de degradação
}

\section{Paulo M. F. Viana}

As geomembranas têm sido largamente utilizadas em barreiras impermeabilizantes nas últimas décadas. Além da boa performance do material, apresentada em situações extremas, o custo-benefício do produto tem favorecido a sua aplicação em diversas obras. Este artigo apresenta os principais aspectos relacionados aos agentes de degradação das geomembranas. Ainda, exemplifica intervalos de confiança e critérios para controle do produto.

Palavras-chave: Geomembranas; PVC; PEAD; Barreiras impermeabilizantes.

The geomembranes has been widely used in barriers in recent decades. Besides the good performance of the material presented in extreme situations the cost effectiveness of the product has promoted their application in several situations. This paper presents the main aspects related to the agents of degradation of geomembranes. Also, exemplifies confidence intervals and criteria for control of product.

Keywords: Geomembranes; PVC; PEAD; Barriers. 


\section{Introdução}

Nas últimas décadas a Engenharia, especialmente a Geotécnica, tem experimentado um crescimento admirável com relação à investigação, concepção, execução e acompanhamento de obras. Certamente, considerando os materiais empregados, os geossintéticos representam o maior avanço, exatamente por auxiliarem na redução da prática do experimentalismo empírico tão presente em obras de características singulares como as de solos naturais. Desde 1971, quando produzido o primeiro geossintético brasileiro, até agora, o número de aplicações e a diversidade de produtos revelaram a potencialidade da utilização destes materiais. Os geossintéticos podem ser basicamente utilizados em obras de controle de erosão, drenagem, filtração, reforço, separação, impermeabilização e proteção e atualmente têm-se registrado mais de 400 produtos e cerca de uma centena de aplicações ${ }^{1,2}$.

O geossintético - G é um produto polimérico (sintético ou natural) industrializado, desenvolvido para aplicação em obras geotécnicas. A família dos geossintéticos reúne Geobarras - GBA, Geocélulas - GL, Geocompostos GC, Geospaçadores - GSP, Geoexpandidos, Geofôrmas, Geogrelhas - GG, Geomantas - GA, Geomembranas GM, Georrede, GN, Geotêxteis-GT, Geotiras e Geotubos. A norma ABNT/NBR 12.5533 apresenta a classificação, definições e siglas dos geossintéticos descritos.

Barreiras impermeabilizantes são geralmente empregadas em obras de canais de adução e irrigação, áreas de disposição de resíduos, lagoas de contenção, retenção, esporte e lazer, criação, tanques de armazenamento, silos subterrâneos, praias artificiais, confinamento de áreas contaminadas, etc. Nestas obras, têm-se substituído soluções tradicionais como camadas compactadas de argila, concreto, asfalto ou até mantas impregnadas com substâncias químicas por Geomenbranas - GM e/ou Geocompostos Argilosos - GCLs. Além de evitar trincas e grandes deslocamentos de materiais, os geossintéticos podem apresentar inúmeras vantagens quando aplicados adequadamente. As principais vantagens do emprego de GMs como barreiras impermeabilizantes são: redução da espessura das camadas, facilidade de aplicação devido a geometria, elevada flexibilidade, possibilidade de instalação em regiões onde os depósitos de argila escassos, elevado controle de qualidade durante a fabricação, facilidade de substituição quando apresentar danos e custo competitivo ${ }^{4,5,6}$.

Quando utilizados como barreiras impermeabilizantes os geossintéticos devem apresentar resistência e durabilidade frente a solicitações de natureza química, biológica, física e mecânica. A ação preventiva da barreira impermeabilizante aliada à capacidade de impedir a migração de fluídos, líquidos ou gases pode ser comprometida quando os requisitos de estabilidade externa e interna não são atendidos. Geossintéticos instalados nesta situação devem apresentar resistência de interface mínima para garantir que os estados limites não sejam atingidos. "A baixa aderência entre as camadas pode causar a ruptura ao longo das interfaces solo-geossintético ou geossintético-geossintético"2. O funcionamento inadequado proveniente de uma ruptura de interface interna ou externa pode danificar o sistema de impermeabilização levando a: perdas de materiais, redução excessiva da camada de proteção, deslizamento do material de cobertura, problemas de condutividade hidráulica, perda da resistência, ruptura da camada de proteção com conseqüente vazamento e danos ambientais, etc. Deste modo é de fundamental importância conhecer os principais agentes de degradação que ativam o processo de eficiência do produto. Este trabalho apresenta uma revisão literária sobre esta temática.
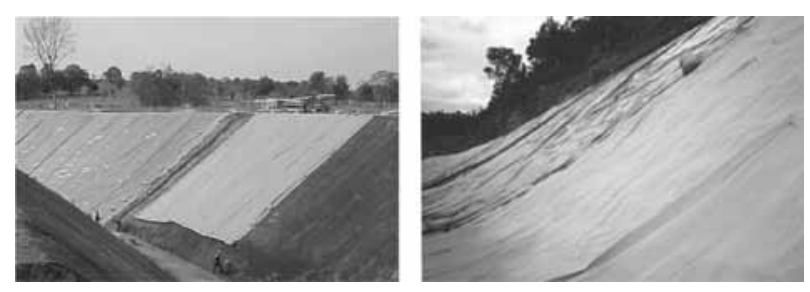

Figura 1: Instalação de GMs em obras de proteção de canais e aterros sanitários ${ }^{7,8,6}$

\section{AS GEOMEMBRANAS - GMS}

As geomembranas - GM [GMB] representam um dos maiores grupos dos geossintéticos. São produtos bidimensionais de baixíssima permeabilidade $\left(\mathrm{k} \cong 10^{-12}\right.$ $\mathrm{cm} / \mathrm{s}$ ) basicamente formadas por materiais termoplásticos, elastômetros e asfálticos, cuja matérias-primas mais utilizadas são o PVC (Policloreto de vinila), o PE (Polietileno de baixa - PEBD e alta densidade - PEAD) e 
o PP (Polipropileno) e ainda como revestimento externo, o PECS (Polietileno corossulfonado), o IIR (Copolímero isobutileno-isopreno), o PEC (polietileno clorado), o EDPM (Copolímero-etileno-propileno) e o neoprene. A principal função da GM é a impermeabilização (bloqueio e desvio de fluidos) $)^{1,3}$. As principais GM são constituídas de HDPE (PEAD) e PVC, geralmente podem apresentar a superfície lisa ou rugosa, variando em função da necessidade o padrão da rugosidade. A Figura 2 apresenta os principais tipos de GM.
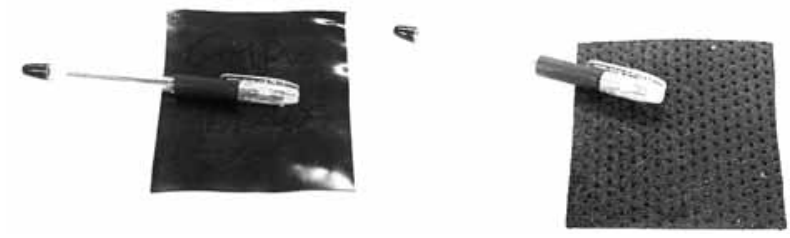

Figura 2: Tipos de geomembrana

As primeiras utilizações das GMs foram em revestimento de piscinas - 1933 (PVC) e como barreiras impermeabilizantes de estradas e canais em 1939 e 1955 - (PE) $)^{10,11,12}$. Certamente os excelentes desempenhos das aplicações iniciais impulsionaram o desenvolvimento das GMs. A primeira aplicação de GM no Brasil foi em 1983, na obra da Alcoa Alumínio S/A - São Luiz/ MA. Nesta obra foram instaladas mais de $500 \mathrm{mil} \mathrm{m}^{2}$ para impermeabilização de lagoas de rejeito de bauxita. Obras relevantes de revestimento impermeabilizante em Sorocaba/SP - 1997 (tanques de água de incêndio), em Itiquira/MT - 2002 (canais de adução) e em Barbacena/ PA - 2002 (Bacias de deposição de rejeitos) são citadas1. A GM pode ainda ser protegida por uma camada de geotêxtil não-tecido como utilizado na AHE Itiquira (Dreno de Base) - Mato Grosso/MT (100 mil m²), no aterro de Disposição de Resíduos Industriais da empresa ICI do Brasil - Paulínia/SP (1,6 mil m²) e no sistema de impermeabilização de passagens subterrâneas, como a instalação próxima ao Shopping Rio-Sul - Rio de Janeiro/RJ (8,1 $\left.\mathrm{mil} \mathrm{m}^{2}\right)$.

Finalmente, para correta aplicação de GMs em obras de barreiras impermeabilizantes deve-se verificar: a resistência das interfaces internas e externas, produção (composição molecular, espessura, densidade, índice de fluidez, dureza e estabilidade dimensional), transporte, instalação, serviço e desempenho (resistência a tração, punção - estática e dinâmica, rasgo, resistência química e durabilidade, permeabilidade adequada, etc). Além disso, a GM deve apresentar resistência à radiação ultravioleta, química, degradação biológica, térmica, resistência a soldas e a fissuração.

\section{PERFORMANCE - AGENTES DE DEGRADAÇÃO}

Para que o produto seja utilizado em uma determinada obra é necessário avaliar a sua performance frente aos agentes que podem causar perda de funcionalidade e desempenho durante a instalação e a longo prazo. As etapas que precedem à especificação do produto segundo normalizações específicas são: a caracterização, a avaliação de desempenho e durabilidade4. Na etapa de caracterização são realizados ensaios de espessura, densidade, massa por unidade de área, tração, calorimetria diferencial de varredura - DSC, Análise termogravimétrica - TGA, análise termomecânica TMA, índice de fusão - MFI, teor de solventes, dureza, extraíveis, teor de cinzas, tempo de oxidação - OIT, teor de negro de fumo - PE e dispersão de negro de fumo PE. Nesta fase o material deve apresentar valores dentro de limites aceitáveis para a aplicação.

A performance do material é avaliada mediante ensaios normalizados de laboratório de desempenho e durabilidade. Os ensaios de desempenho são: resistência ao rasgo, estouro e puncionamento, cisalhamento, fluência, expansão térmica, estabilidade dimensional e permeabilidade (água e vapor). Um outro aspecto que deve ser considerado é a resistência da emenda, que neste caso deve ser avaliada segundo os ensaios: Ponto de escoamento mecânico, cisalhamento, descolamento, pressurização, aplicação de ar, vácuo, impedância e ressonância ultra-sônica. Finalmente, em relação à durabilidade, os principais ensaios são: abrasão, exposição à intempérie, envelhecimento térmico e por ozônio, exposição ao calor, radiação UV, resistência química e biológica e fissuramento sob tensão - PE14.

Os principais agentes de degradação das GMs são: a) Mecânicos - devido ações ambientais ativas - vento, sobrecargas acidentais, cargas estáticas e dinâmicas que provocam rasgo, punção, estouro, cisalhamento, fluência, expansão, abrasão, fissuramento (stress cracking - SC), descolamento e escoamento mecânico, 
b) Térmicos - variações de temperatura que provocam expansão, fluência, perda de estabilidade, amolecimento, enrijecimento e c) Químicos e Biológicos - radiações diversas, radiação UV, raios infravermelhos, volatização, deslaminação, ozônio, oxidação e ações químicas diversas (ex: biodegradação de microorganismos) que provocam envelhecimento, fissuramento, descoloração, enrijecimento, etc. Estas ações podem ser intensificadas em função da interação com o sistema devido fatores de operação, como exemplo, quando a manta é instalada em um sistema agressivo e com recalques diferenciais. Em relação aos agentes biológicos, estes podem contribuir para a intensificação de outros agentes, como exemplo, o crescimento de raízes ou ações de animais que pode favorecer a perda da resistência estrutural da manta, nestes casos, é de fundamental importância avaliar de modo específico a intensidade de cada evento.

Como exemplo, apresenta-se na Figura 3 a variação do módulo secante (rigidez) com o tempo para 100\% de deformação de amostras exumadas de GM de PVC de $0,8 \mathrm{~mm}$. Apesar de atender a especificação normativa, para este caso, de $5,3 \mathrm{kN} / \mathrm{m}$ é evidente a variação sofrida no material devido aos agentes de degradação.

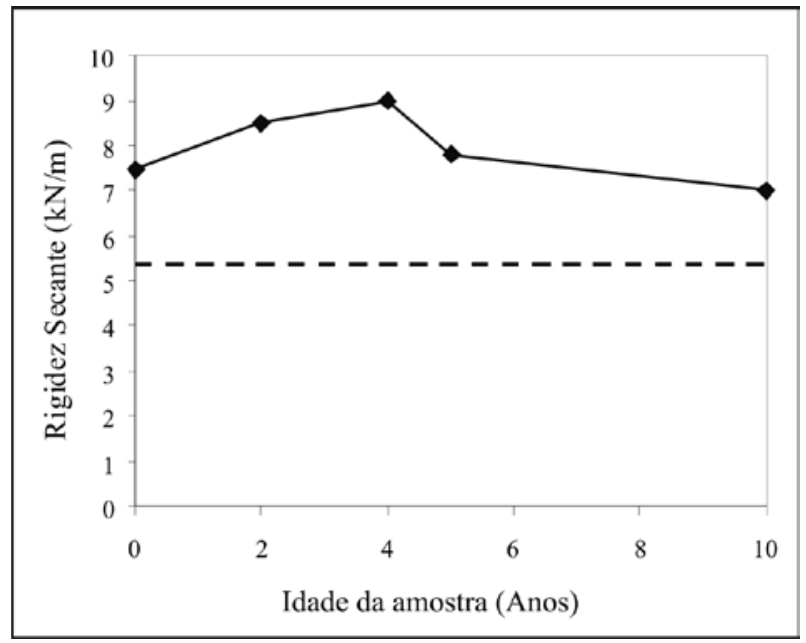

Figura 3: Variação do módulo secante (rigidez - direção longitudinal) com o tempo para $100 \%$ de deformação de amostras exumadas de GM de $\mathrm{PVC}^{15}$.

\section{INTERVALOS DE CONFIANÇA E CRITÉRIOS DE CONTROLE}

Os intervalos de confiança são diferenças aceitáveis para as principais variáveis de performance do material após exposição a um agente de degradação. A Tabela 14 apresenta intervalos de confiança das principais propriedades das GMs (PEAD e PVC) após exposição a resíduos e/ou lixívias.

Tabela 1: Valores limites de diferentes ensaios de GM incubadas ${ }^{4}$.

\begin{tabular}{lccc}
\hline Propriedades & & Resistente & Não - Resistente \\
PVC & & & \\
Taxa de permeabilidade & $\mathrm{g} / \mathrm{m}^{2} / \mathrm{h}$ & $<0,9$ & $<0,9$ \\
Peso & $\%$ & $<10$ & $>10$ \\
Volume & $\%$ & $<10$ & $>10$ \\
Resistência à tração & $\%$ & $<20$ & $>20$ \\
Alongamento na ruptura & $\%$ & $<30$ & $>30$ \\
Módulos (100 e 200 \%) & $\%$ & $<30$ & $>30$ \\
Resistência a dureza & $\%$ & $<10$ & $>10$ \\
\hline PEAD & & & \\
Taxa de permeabilidade & $\mathrm{g} / \mathrm{m}^{2} / \mathrm{h}$ & $<0,9$ & $=0,9$ \\
Peso & $\%$ & $<2$ & $=2$ \\
Volume & $\%$ & $<1$ & $=1$ \\
Resistência à tração & $\%$ & $<20$ & $=20$ \\
Alongamento no escoamento & $\%$ & $<30$ & $=30$ \\
Módulos & $\%$ & $<30$ & $=30$ \\
Resistência a o rasgo & $\%$ & $<20$ & $=20$ \\
Resistência ao puncionamento & $\%$ & $<30$ & $=30$ \\
\hline & & & \\
\hline & $\%$ & \\
\hline
\end{tabular}

Associado ao controle de laboratório é de fundamental importância garantir que durante a fabricação os produtos utilizados como matérias-primas atendam as especificações normativas. Ainda, deve-se garantir que durante instalação e vida útil sejam realizados ensaios de controle de qualidade/inspeção. Os principais métodos empregados para caracterizar as matérias-primas empregadas, bem como, as vantagens e desvantagens de cada um são apresentados em literatura específica14.

Um rigoroso controle de qualidade dos produtos durante a fabricação (CQF) e instalação (CQI), bem como a garantia de qualidade da fabricação e instalação (GQF e GQI) é de fundamental importância4. Além da garantia e do controle deve-se garantir que as propriedades do material especificado sejam aquelas presentes no produto entregue na obra, ou seja, que produto seja conforme o 
especificado. A conformidade é um importante aspecto do projeto e deve atender a uma frequência de ensaios pré-definida pelo responsável técnico.

Exemplos de especificação mínima de ensaios para atender os requisitos de conformidade, bem como, a relação de propriedades e parâmetros para o controle de instalação podem ser obtidos na literatura ${ }^{14}$.

\section{Conclusões}

Durante a fabricação, transporte, instalação e vida útil as GMs podem ser afetadas por diversos fatores que podem contribuir para redução da sua performance. O correto entendimento dos agentes de degradação predominantes é de fundamental importância para definir as características do material a ser utilizado.

Os agentes de degradação são: Mecânicos, térmicos, químicos e biológicos. A atuação e associação destes agentes irão produzir efeitos noviços que devem ser considerados de modo que o material atenda os critérios de qualidade e de garantia necessários para o seu bom desempenho.

É de fundamental importância considerar intervalos de confiança para aplicações específicas de modo que seja garantida a boa utilização do material. Deste modo, o acompanhamento mediante ensaios de laboratório e campo é essencial.

Neste contexto, o material deve apresentar os requisitos de conformidade para que suas propriedades sejam garantidas durante a instalação.

\section{BIBLIOGRAFIA}

1. Aguiar, P.R.; Vertamatti, J.C.; ABINT. Manual Brasileiro de Geossintéticos, Edgard Blücher: São Paulo, 01, 2004.

2. Viana, P.M.F.; Palmeira, E.M.; Internal Report, Graduate Programme of Geotechnics, University of Brasilia, Brazil. 2008, 57p.

3. Associação Brasileira de Normas Técnicas; Geossintéticos - Terminologia, ABNT: São Paulo, disponível em: http://www.abnt.org.br. 2003.

4. Koerner, R.M.; Designing with Geosynthetics, 4 ed., Prentice Hall: New Jerzey, 1998.

5. Viana L.; H.N.; Palmeira, E.M.; Seminário de
Qualificação em Geotecnia, UnB. 2006.

6. Jennyever (HK) INT'L TRADING CO.; Bentonite Geosynthetic Clay Liners. Disponível em: www. jennyever.com/products $/ \mathrm{gcl} / \mathrm{gcl} . \mathrm{htm}$ consultado em: 07/08/2008.

7. Bidim Informa; Informativo - Apresentação. BIDIM: São Paulo, 4, 2003.

8. Bidim Informa; Informativo n.55. BIDIM: São Paulo, 4, 2004.

9. Bell, J.R.; Yoder, E.J.; Proceedings of the Highway Research Board, HRB: Washington, 1957, 36, 713.

10. Staff, C.E.; Proceedings of the I International Conference on Geomembranes. ICG: Denver, 1, 5, 1984

11. Hawkins, G.; Proceedings of the I International Conference on Geomembranes. ICG: Denver, 1, 15, 1984

12. Sharma, H.D.; Lewis, S.P.; Waste Containment Systems, Waste Stabilization and Landfills: Design and Evaluation, Jonh Wiley \& Sons, 558, 1994.

13. Lodi, P.C.; Tese de Doutorado, Universidade de São Paulo, Brasil, 2003.

14. Newman, E.J.; Stark, T.D.; Geosynthetics International, 2009, 16, (2), 1072-6349, 12p.

\section{Paulo Márcio Fernandes Viana $^{* 1}$}

1 Curso de Engenharia Civil. Universidade Estadual de Goiás UnUCET/UEG. Rodovia BR153, Arco Verde, 750001-970 Anápolis, GO, Brasil.

*e-mail: paulo.viana@ueg.br

Telefone: $+55(62)$ 3328-1160 